\title{
Selbstmanagement in der Blutgerinnungskontrolle
}

\author{
Höhere Lebensqualität und Einsparungspotential sind möglich
}

Mehr als 40.000 Menschen müssen in Österreich lebenslang blutgerinnungshemmende Medikamente einnehmen, um Komplikationen wie Schlaganfall oder Embolien zu vermeiden. Die enge therapeutische Breite der am häufigsten verwendeten Substanzklasse, der Vitamin-K-Antagonisten, erfordert jedoch eine regelmäßige Kontrolle der Blutgerinnung. Obwohl Point-of-Care Geräte für ein effizientes Selbstmanagement verfügbar sind und sich etwa 40 Prozent der Patienten dafür eignen würden, nützen nur zehn Prozent der Betroffenen diese Methode. Studien haben gezeigt, dass Patienten mit Selbstmanagement bessere therapeutische Gerinnungswerte aufweisen, dadurch eine höhere Lebensqualität gewinnen und die Kosten für das Gesundheitssystem mittelfristig niedriger sind als bei der regelmäßigen Fremdkontrolle. Medizinische und gesundheitsökonomische Experten präsentierten Ende Juni in Wien unter dem Titel „Mündige Patienten zahlen sich aus" aktuelle Ergebnisse.

Orale Antikoagulantien können in der Primär- und Sekundärprophylaxe eine relative Risikoreduktion bis zu 75 Prozent erzielen, stellte Dr. Benjamin Dieplinger, Zentrallabor der Barmherzigen Brüder und Barmherzigen Schwestern, Linz, fest. Während es in der Primärprophylaxe um die Vermeidung von Komplikationen bei Vorhofflimmern oder nach Implantation künstlicher Herzklappen geht, ist die Sekundärprophylaxe vor allem nach venös thrombo-embolischen Ereignissen (VTE) indiziert. Derzeit am weitesten verbreitet seien Vitamin K-Antagonisten wie Marcoumar, deren Wirkung jedoch regelmä- ßig kontrolliert werden muss, was derzeit im überwiegenden Anteil vom niedergelassenen Arzt oder in der Spitalsambulanz alle vier bis sechs Wochen durchgeführt werde. Der Vorteil des Selbstmonitorings: Die Kontrolle wird - nach intensiver Einschulung - vom Patienten selbst wöchentlich durchgeführt. Die höhere Frequenz der Messung führt dazu, dass Abweichungen früher erkannt und entsprechend gegengesteuert werden kann. Liegt der Wert innerhalb einer vordefinierten Abweichung, kann der Patient die Dosis selbst anpassen, liegt sie außerhalb dieses Bereichs muss er den Arzt/die Ambulanz aufsuchen. Vierteljährlich erfolgt darüberhinaus in jedem Fall eine ärztliche Kontrolle. „Jedem Patienten, der dazu physisch und kognitiv in der Lage ist, sollte heute Selbstmonitoring angeboten werden", betonte Dieplinger.

Eine gesundheitsökonomische Studie von Joanneum Research verglich Selbstund Fremdmonitoring und zeigte am Beispiel der Situation im Burgenland und in Kärnten sowohl medizinische Effekte als auch ökonomische Unterschiede. Dabei wurden in einer modellbasierten Analyse auf der Grundlage internationaler Effektivitätsdaten und regionaler Mortalitätsdaten sowie nationaler Kostendaten folgende Komplikationen betrachtet: Ischämischer Insult, hämorrhagischer Insult, transitorisch ischämische Attacke (TIA), Lungenembolie und gastrointestinale Blutung. Zwischen den beiden Gruppen ergaben sich hinsichtlich der Komplikationen unterschiedliche Effektdaten zugunsten des Selbstmanagements gegenüber dem Fremdmonitoring, berichtete Dipl. Ing. Dr. Wolfgang Habacher, Inst. f. Med. Systemtechnik und Gesundheitsmanagement der Joanneum Research ForschungsgesmbH. Weniger Arztbesuche führen zu geringeren laufenden Kosten, hinzu kommen die Folgekosten der Komplikationen. Dies führt dazu, dass sich die höheren Initialkosten beim Selbstmanagement durch Geräteanschaffung und Schulungskosten innerhalb von drei Jahren amortisieren und Selbstmonitoring in den weiteren Jahren gegenüber Fremdmonitoring kostengünstiger ist. Hinzu kommt die höhere Lebensqualität durch die bessere Gerinnungseinstellung und weniger Komplikationen: „Selbstmanagement", so Habacher, „führt zu 1,3 gewonnenen ,qualitätskorrigierten Lebensjahren pro Patient (QALYs) während Fremdmonitoring in einer geringeren Anzahl an QALIs resultiert."

Ein Grund für den geringen Anteil an Patienten, die in Österreich ihre Blutgerinnung im Selbstmanagement kontrollieren, liegt in der zaghaften und je nach Bundesland unterschiedlichen Erstattung der Kosten. Würde man allerdings den Anteil, wie in Deutschland, auf 20 Prozent erhöhen, wären - konservativ berechnet Einsparungen für das Gesundheitssystem in der Höhe von 1,2 bis 3,75 Millionen Euro möglich, stellt Prof. Dr. Thomas Schröck, Gesundheitsökonom, fest. Die neuen oralen Antikoagulantien, deren Vorteil auch darin liegt, dass sie ohne Gerinnungskontrolle auskommen, dürften in absehbarer Zeit aus Kostengründen eher restriktiv eingesetzt werden, vermutet Dieplinger.

Quelle: Roche Diagnostics Pressefrühstück „Mündige Patienten zahlen sich aus. Aktuelle Studienergebnisse: Patientenselbstmanagement in der Blutgerinnungskontrolle ist die kostengünstigste und effektivste zugelassene Therapieform.", 28. Juni 2012, Wien 